Supporting Information

\title{
High dielectric constant and low percolation threshold in Poly(vinylidene fluoride) Composite Films Containing Mechanochemically Synthesized $\mathrm{BaSnF}_{4}$ Nanowires
}

Chang-Yeol Cho ${ }^{\dagger}$, Seung-Hyun Kim ${ }^{\dagger} \ddagger$, Jong-Wook Ha ${ }^{\dagger}$, Hong Suk Kang ${ }^{\dagger}$, Eun-Ho Sohn ${ }^{\dagger}$, In Jun Park ${ }^{\dagger}$, Gi-Ra Yi* and Sang Goo Lee ${ }^{\dagger}, *$

†Interface Materials and Chemical Engineering Research Center, Korea Research Institute of Chemical Technology, Daejeon 34114, Republic of Korea

School of Chemical Engineering, Sungkyunkwan University, Suwon 16419, Republic of Korea

*E-mail : sgoo@krict.re.kr 


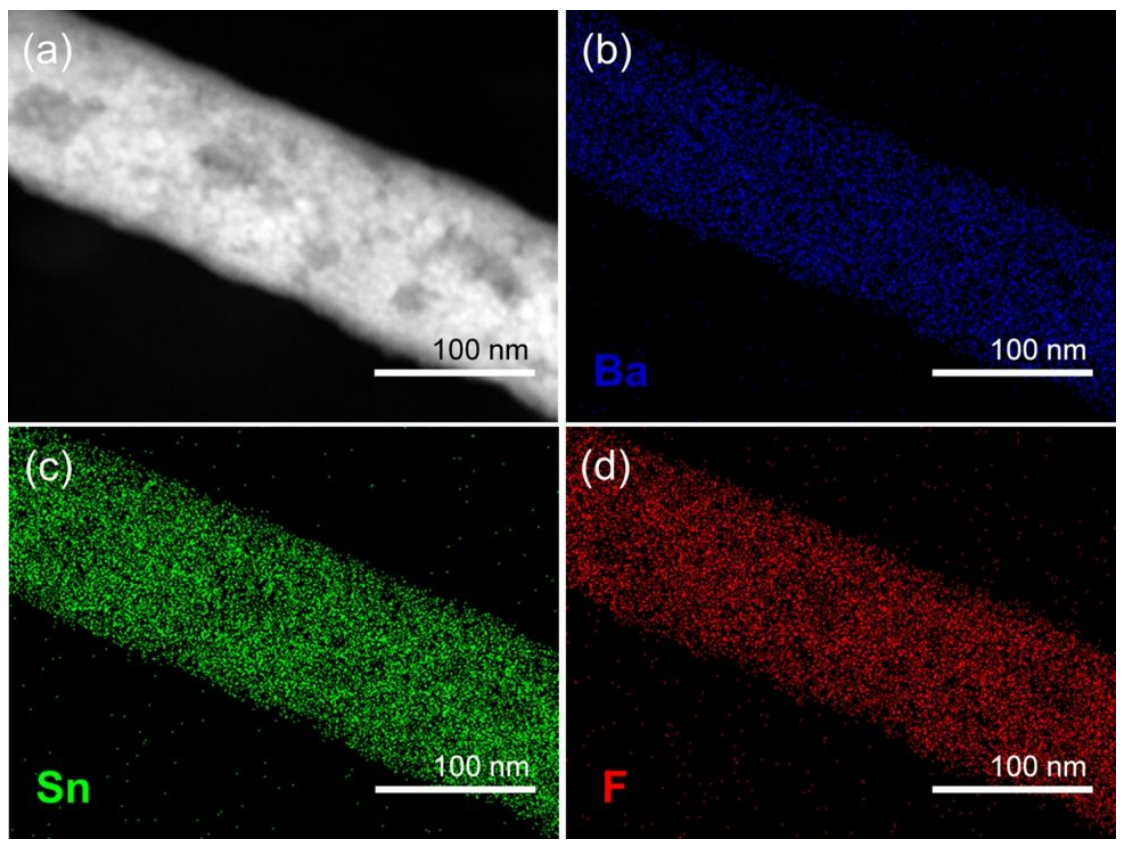

Figure S1. TEM image of (a) $\mathrm{BaSnF}_{4}$ nanowire. EDS mapping of (b) $\mathrm{Ba}$, (c) Sn and (d) $\mathrm{F}$ element on the TEM image of the $\mathrm{BaSnF}_{4}$ nanowire. 
Figure S2. SEM image of $\mathrm{BaSnF}_{4}$ nanoparticles. 

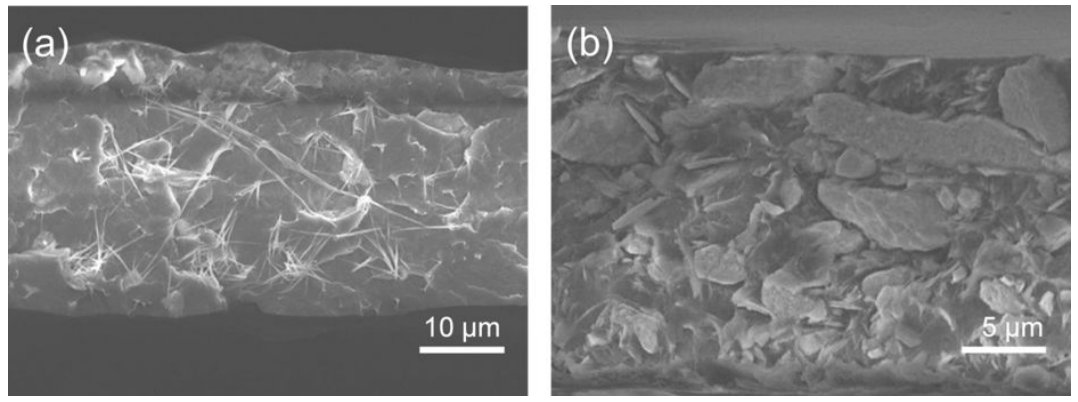

Figure S3. SEM images of the cross-section of (a) $\mathrm{BaSnF}_{4}$ NW/PVDF and (b) $\mathrm{BaSnF}_{4}$ a-NP/PVDF composite films. 
Table S1. Dielectric properties and breakdown strength of various PVDF nanocomposites reported in the literature

\begin{tabular}{|c|c|c|c|c|c|c|c|}
\hline $\begin{array}{l}\text { Polymer } \\
\text { Matrix }\end{array}$ & $\begin{array}{l}\text { Inorganic } \\
\text { Filler }\end{array}$ & $\begin{array}{c}\text { Total filler } \\
\text { concentrati } \\
\text { on } \\
\text { (vol\%) }\end{array}$ & $\begin{array}{c}\text { Frequenc } \\
y \\
(\mathrm{~Hz})\end{array}$ & $\begin{array}{c}\text { Dielectri } \\
\text { C } \\
\text { constant }\end{array}$ & $\begin{array}{c}\text { Loss } \\
\text { tangent }\end{array}$ & $\begin{array}{c}\text { Breakdown } \\
\text { strength } \\
(\mathrm{MV} / \mathrm{m})\end{array}$ & Ref \\
\hline PVDF a) & $\mathrm{BaSnF}_{4} \mathrm{NW}$ & 10 & $10^{3}$ & 20.5 & 0.095 & 389.2 & $\begin{array}{c}\text { our } \\
\mathrm{s}\end{array}$ \\
\hline PVDF & $\mathrm{BaSnF}_{4} \mathrm{NW}$ & 30 & $10^{3}$ & 67 & 0.418 & 291.3 & $\begin{array}{c}\text { our } \\
\mathrm{s}\end{array}$ \\
\hline PVDF & $\mathrm{CaCu}_{3} \mathrm{Ti}_{4} \mathrm{O}_{12} \mathrm{NW}^{\mathrm{d})}$ & 30 & $10^{3}$ & 49 & 0.25 & - & 1 \\
\hline PVDF & $\mathrm{BaTiO}_{3} \mathrm{NW}$ & 30 & $10^{3}$ & 44.3 & 0.042 & - & 2 \\
\hline PVDF & $\mathrm{PbZr}_{\mathrm{x}} \mathrm{Ti}_{1-\mathrm{x}} \mathrm{O}_{3} \mathrm{NW}$ & 30 & $10^{3}$ & 35 & - & 80 & 3 \\
\hline PVDF & $\begin{array}{c}\text { Ag NPe) deposited } \\
\mathrm{BaTiO}_{3}\end{array}$ & 27.3 & $10^{2}$ & 45 & 0.07 & 180 & 4 \\
\hline PVDF & $\begin{array}{c}\mathrm{KTa}_{0.5} \mathrm{Nb}_{0.5} \mathrm{O}_{3} \\
\mathrm{NR}^{\mathrm{h})}\end{array}$ & 30 & $10^{2}$ & 68 & 0.076 & 63 & 8 \\
\hline PVDF-HFPb) & $\mathrm{BaTiO}_{3} \mathrm{NW}$ & 10 & $10^{2}$ & 49 & 0.63 & 100 & 5 \\
\hline PVDF & $\begin{array}{l}\text { PVPf) modified } \\
\left.\mathrm{SrTiO}_{3} \mathrm{NF}^{\mathrm{g}}\right)\end{array}$ & 7.5 & $10^{3}$ & 14.9 & 0.015 & 290 & 6 \\
\hline PVDF & $\begin{array}{c}\text { PVP modified } \\
\mathrm{BaZr}_{0.3} \mathrm{Ti}_{0.7} \mathrm{O}_{3} \mathrm{NF}\end{array}$ & 7.5 & $10^{3}$ & 18.6 & 0.018 & 290 & 7 \\
\hline PVDF-TrFEc) & $\begin{array}{c}\text { Dopamine } \\
\text { modified } \mathrm{BaTiO}_{3} \\
\mathrm{NF}\end{array}$ & 10.8 & $10^{3}$ & 30 & 0.02 & 204.8 & 9 \\
\hline
\end{tabular}

*a) PVDF: poly(vinylidene fluoride), b) PVDF-HFP: poly(vinylidene fluoride-hexafluoropropylene), c) PVDF-TrFE: poly(vinylidene fluoride-trifluoroethylene), d) NW: nanowire, e) NP: nanoparticle f) PVP: polyvinylpyrrolidone, g) NF: nanofiber h) NR: nanorod 


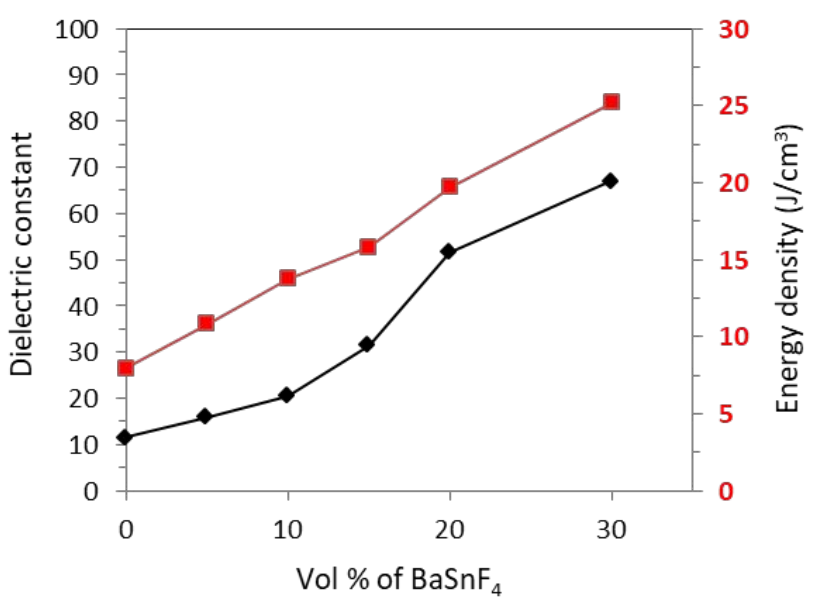

Figure S4. Dielectric constant and energy density of $\mathrm{BaSnF}_{4} \mathrm{NW/PVDF}$ composite measured at $10^{3} \mathrm{~Hz}$ and room temperature as a function of $\mathrm{BaSnF}_{4}$ volume fraction. 


\section{References}

(1) Tang, H.; Zhou, Z.; Bowland, C.C. and Sodano, H.A. Synthesis of Calcium Copper Titanate $\left(\mathrm{CaCu}_{3} \mathrm{Ti}_{4} \mathrm{O}_{12}\right)$ Nanowires with Insulating $\mathrm{SiO}_{2}$ Barrier for Low Loss High Dielectric Constant Nanocomposites. Nano energy 2015, 17, 302-307.

(2) Zhou, H.T.Z. and Sodano, H.A. Relationship between $\mathrm{BaTiO}_{3}$ Nanowire Aspect Rato and the Dielectric Permittivity of Nanocomposites. ACS Appl. Mater. Interfaces 2014, 6, 5450.

(3) Tang,H.; Lin,Y and Sodano, H.A. Enhanced Energy Storage in Nanocomposite Capacitors through Aligned PZT Nanowires by Uniaxial Strain Assembly. Adv. Energy Mater. 2012, 2, 469-476.

(4) Liu, H.; Luo, S.; Yu, S.; Ding, S.; Shen, Y.; Sun, R. and Wong, C.P. Flexible $\mathrm{BaTiO}_{3}$ nfAg/PVDF with High Dielectric Constant and Energy Density. IEEE Trans. Dielectr. Electr. Insul. 2017, 24, 757-763.

(5) Chen, G.; Yang, W.; Lin, J.; Wang, X.; Li, D.; Wang, Y.; Liang, M.; Ding, W.; Li, H. and Lei, Q. Geometrical Shape Adjustment of $\mathrm{KTa}_{0.5} \mathrm{Nb}_{0.5} \mathrm{O}_{3}$ Nanofillers for Tailored Dielectric Properties of $\mathrm{KTa}_{0.5} \mathrm{Nb}_{0.5} \mathrm{O}_{3} / \mathrm{PVDF}$ Composite. J. Mater. Chem. C 2017, 5, 8135-8143.

(6) Feng, Y.; Li, W.L.; Hou, F.Y.; Yu, Y.; Cao, W.P.; Zhang, T.D. and Fei, W. D. Enhanced Dielectric Properties of PVDF-HFP/BaTiO 3 -nanowire Composites Induced by Interfacial Polarization and Wire-shape. J. Mater. Chem. C 2015, 3, 1250-1260. 
(7) Liu, S. and Zhai, J. Improving the Dielectric Constant and Energy Density of Poly(vinylidene fluoride) Composites Induced by Surface-modified $\mathrm{SrTiO}_{3}$ Nanofibers by Polyvinylpyrrolidone. J. Mater. Chem. A 2015, 3, 1511-1517.

(8) Liu, S.; Xue, S.; Xiu, S; Shen, B. and Zhai, J. Surface-modified $\mathrm{Ba}\left(\mathrm{Zr}_{0.3} \operatorname{Ti}_{0.7}\right) \mathrm{O}_{3}$ Nanofibers by Polyvinylpyrrolidone Filler for Poly(vinylidene fluoride) Composites with Enhanced Dielectric Constant and Energy Storage Density. Scientific reports 2016, 6, 26198.

(9) Song, Y.; Shen, Y.; Liu, H.; Lin, Y.; Li, M. and Nan, C.W. Enhanced Dielectric and Ferroelectric Properties Induced Dopamine-modified $\mathrm{BaTiO}_{3}$ Nanofibers in Flexible Poly(vinylideneFluoride-trifluoroethylene) Nanocomposites. J. Mater. Chem. 2012, 22, 8063-8068. 